\title{
Gravity-induced instability and gauge field localization
}

\author{
K. Farakos *and P. Pasipoularides ${ }^{\dagger}$ \\ Department of Physics, National Technical University of Athens \\ Zografou Campus, 15780 Athens, Greece
}

\begin{abstract}
The spectrum of a massless bulk scalar field $\Phi$, with a possible interaction term of the form $\mathcal{L}_{\text {int }}=-\xi R \Phi^{2}$, is investigated in the case of RS-geometry [1]. We show that the zero mode for $\xi=0$, turns into a tachyon mode, in the case of a nonzero negative value of $\xi(\xi<0)$. As we see, the existence of the tachyon mode destabilizes the $\Phi=0$ vacuum, against a new stable vacuum with nonzero $\Phi$ near the brane, and zero in the bulk. By using this result, we can construct a simple model for the gauge field localization, according to the philosophy of Dvali and Shifman (Higgs phase on the brane, confinement in the bulk).
\end{abstract}

\section{Introduction}

It is well known that theories which are believed as fundamental, i.e. the string theory, are formulated in multidimensional spaces. For this reason there has been a great interest in field theory models with more than three spatial dimensions. The standard way to retain four-dimensional physics in multidimensional-models is to assume that the extra dimensions are compactified to a large scale $M$. However, in resent years, there is an alternative scenario which has attracted attention. This scenario is based on the idea according to which we are trapped in a submanifold with three spatial dimensions (brane world) that is embedded in a fundamental multi-dimensional manifold (bulk). A new feature of this scenario, is that it allows the extra dimensions to be large or even infinite.

In this paper we will concentrate our attention on the interesting case of the RS2-brane world scenario of Ref. [1. In this variation of the brane world scenario, we have a single brane with a positive energy density (the tension $\sigma$ ) and a negative, fine-tuned, five dimensional cosmological constant $\Lambda$. This model implies a non-factorizable space time geometry of the form of $A d S_{5}$ around the brane (see Eq. (5) below). The four-dimensional particles, in this model, are expected to be gravitationally trapped on the brane. We see in Refs. [1, 3] that gravitons and scalar fields exhibit a normalizable zero mode plus a continuous spectrum ${ }^{1}$.

\footnotetext{
*kfarakos@central.ntua.gr

†paul@central.ntua.gr

${ }^{1}$ In this case there is no mass gap. However, the continuous modes interact very weakly with the four dimensional matter (zero mode). In this way the continuous modes do not affect the low energy four dimensional physics
} 
Unfortunately there is no normalizable zero mode in the case of fermions and gauge fields ${ }^{2}$, for the RS2-model, see Ref. 3].

According to the above discussion, for achieving localization of the fermion or gauge field we must invoke an alternative scenario or an alternative mechanism. There are several variation of the brane world scenario, with different geometries than that of the RS2-model (different warp factors) or with more than five dimensions, or even in flat space time with topological defects, where the fermion or gauge field localization has been achieved, see Refs. [4, 5, 6] and references there in. However, as it is emphasized in Ref. [4, 7], the most difficult task is the massless non-Abelian gauge field localization on the brane, as any acceptable mechanism for this must preserve the charge universality.

A non gravitational mechanism, which seems to solve this problem in a clever way, is the Dvali-Shifman mechanism of Ref. [2. This mechanism is based on the idea of the construction of a gauge field model which exhibits a non-confinement phase on the brane and a confinement phase on the bulk. Thus gauge fields, and more generally fermions and bosons with gauge charge (see Ref. 4]), can not escape into the bulk unless we give them energy greater than the mass gap $\Lambda_{G}$, which emerges from the nunpurturbative confining dynamics of the gauge field model in the bulk. For criticism or a better insight on this mechanism see Refs. 4, 7, 8, 9]. Also, the Dvali-Shifman mechanism in the case of conformal matter has been studied in Ref. 10.

From the point of view of lattice, it is worth to note that there are some models with anisotropic couplings, in multi-dimensional spaces, that exhibit a new phase, the layer phase as it is named. The main feature of the layer phase is a non-confinement phase along the layer combined with a confinement along the extra dimensions. The idea of the layer phase was originated by Fu and Nielsen, in Ref. [11. Recent research aiming at the investigation of the layer phase for several models with anisotropic coupling, as a way to achieve nonAbelian gauge field localization, can be found in Refs. [12, 13, 14, 15. Particularly, in Ref. 14] the anisotropic $\mathrm{SU}(2)$ adjoint Higgs model is analyzed. In addition, the $\mathrm{U}(1)$ model in the RS-background is analyzed in Ref. 13]. We see that the lattice results indicate a layer phase for both of these models.

In this paper we aim at the construction of a model where the Dvali-Shifman mechanism is triggered by the geometry of the multidimensional space time, and not by an auxiliary neutral scalar field which forms a kink topological defect toward the extra dimension, as is done in the original paper of Ref. [2]. For this we have considered an SU(2) gauge field model with a scalar triplet, in the RS-geometry. In this model we have included a possible interaction term of the form $\mathcal{L}_{\text {int }}=-\xi R \Phi^{2}$, see Refs. [16, 17, 18, where $\xi$ is a dimensionless numerical factor. In section 4 we study how the spectrum of the scalar field is modified when this additional interaction turns on. We see that the zero mode, for $\xi=0$, turns into a tachyon mode (a normalizable bound state with negative energy), in the case of a nonzero negative value of $\xi(\xi<0)$. The tachyon mode implies an instability of the $\Phi=0$ vacuum against a new stable vacuum for the scalar field, which is described by a $\Phi(z)$ configuration with nonvanishing value for $\Phi$ near the brane ( $z$ is the extra dimension). Thus, in the background of this stable configuration for the scalar field, the gauge symmetry is valid in the bulk (confinement phase), but it is spontaneously broken on the brane (non-confinement

\footnotetext{
${ }^{2}$ There is a normalizable bound state for fermions only if the brane tension in negative (or $\sigma<0$ ). Gauge fields are localized neither on a brane with positive tension nor on a brane with negative tension.
} 
phase).

It is worth to note that in Refs. [18, 19] the same non minimally coupled model is considered, for four dimensions in the case of de Sitter space-time. It is obtained that for a specific range of values of $\xi$ the scalar field is rendered unstable, and this result have straightforward implications to the cosmological constant problem. The philosophy of our paper is quite closely to that of the previous works of Refs. [18, 19]. However there are some important differences, as in the present work we study the five dimensional space-time in the present of the RS-metric, and we use our results in order to argue for the localization of the gauge fields on the brane.

\section{Gravity-induced instability for the scalar field vac- uum $\Phi=0$}

We consider an $\mathrm{SU}(2)$ gauge field model with a scalar triplet $\Phi^{c}(c=1,2,3)$, in the adjoint representation, in the presence of gravity. The action of this model is:

$$
S=\int d^{5} x\left(\mathcal{L}_{\text {gravity }}+\mathcal{L}_{\text {gauge }}+\mathcal{L}_{\text {scalar }}\right)
$$

The volume element is $d^{5} x=d^{4} x d x_{4}$, where $x_{4}$ is the infinite extra dimension. In what will follow we set $x_{4}=z$.

The Lagrangian for gravity is chosen to be identical with that of the Randall-Sundrum model [1], which is given by the equation

$$
\mathcal{L}_{\text {gravity }}=-\frac{1}{16 \pi G_{5}} \sqrt{|g|} R+\Lambda \sqrt{|g|}+\sigma \delta(z) \sqrt{\left|g^{(\text {brane })}\right|}
$$

where $\sigma$ is the brane tension, $\Lambda$ is the five-dimensional cosmological constant, and $G_{5}$ is the five-dimensional Newton 's constant. In addition $R$ is the five dimensional Ricci scalar, $g$ is the determinant of the five dimensional metric tensor $g_{M N}(M, N=0,1, \ldots, 4)$, and $g^{\text {brane }}$ is the determinant of the metric tensor on the brane, which is defined by the equation $g_{\mu \nu}^{(\text {brane })}=g_{\mu \nu}(z=0)(\mu, \nu=0,1, \ldots, 3)$. For the signature of the metric $g_{M N}$ we adopt the convention $(+,-,-,-,-)$, and for the sign of the curvature tensor we adopt the convention of Ref. [16] $\left(R_{\beta \gamma \delta}^{\alpha}=\partial_{\delta} \Gamma_{\beta \gamma}^{\alpha}-\ldots\right)$.

The Lagrangians for the gauge field and the scalar field, in curved space-time, are

$$
\begin{aligned}
\mathcal{L}_{\text {gauge }} & =-\frac{1}{4} \sqrt{|g|} F_{M N}^{c} F_{K L}^{c} g^{M K} g^{N L} \\
\mathcal{L}_{\text {scalar }} & =\sqrt{|g|}\left(\frac{1}{2} g^{M N} D_{M} \Phi_{c} D_{N} \Phi_{c}-V(\Phi)\right)
\end{aligned}
$$

In what follows we assume that $V(\Phi)=\lambda(\Phi \cdot \Phi)^{2}$, or we assume that there is not a mass term. Also we have used the notation $\Phi=\left(\Phi_{1}, \Phi_{2}, \Phi_{3}\right)$ and $\Phi \cdot \Phi=\Phi_{1}^{2}+\Phi_{2}^{2}+\Phi_{3}^{2}$. In addition the covariant derivative in the adjoint representation is $D_{M}=\partial_{M}-i g_{5} A_{M}$ where $A_{M}=A_{M}^{b} T^{b}, T^{b}=-i \epsilon^{b c d}$, and $g_{5}$ is the five-dimensional gauge coupling.

If we chose $\Lambda=\frac{-4 \pi}{3} G_{5} \sigma^{2}$, the corresponding equations of motion of the Lagrangian of Eq. (1) have a classical stable solution of the form

$$
d s^{2}=a^{2}(z)\left(d x_{0}^{2}-d x_{1}^{2}-d x_{2}^{2}-d x_{3}^{2}\right)-d z^{2}, \quad \Phi_{c}=0, \quad A_{M}=0
$$


where $a(z)=e^{-k|z|}$ is the warp factor, with $k=\frac{4 \pi}{3} G_{5} \sigma$.

We aim to investigate the effects of a possible additional interaction term between the scalar field and the gravity, of the form $-\xi R \Phi^{2}$ (see Refs. [16, 17]), where $\xi$ is a dimensionless numerical factor. Then $\mathcal{L}_{\text {scalar }}$ is modified as

$$
\mathcal{L}_{\text {scalar }}=\sqrt{|g|}\left(\frac{1}{2} g^{M N} D_{M} \Phi_{c} D_{N} \Phi_{c}-\frac{1}{2} \xi R(\Phi \cdot \Phi)-V(\Phi)\right)
$$

Note that Eq. (5) remains a solution of the equations of motion even for $\xi \neq 0$.

The spectrum of the massless scalar field, around the stable solution of Eq. (5) for $\xi=0$, consists of a zero-mode plus a continuous tower of states (see for example Ref. [3]). In section 4 we will show analytically that the zero mode for $\xi=0$, turns into tachyon mode (a normalizable bound state with negative energy), in the case of a nonzero negative value of $\xi(\xi<0)$.

The existence of a localized tachyon mode around $z=0$, when we turn on the interaction term $-\xi R \Phi^{2}$, renders the solution of Eq. (5) unstable. Due to the instability, the vacuum expectation value of the scalar field can not be zero everywhere anymore. The scalar field vacuum will be a function of $z$, which form is expected to be similar with that of the tachyon mode, see Eq. (32) in section 4. This suggests a nonzero value for $\Phi_{3}(z)$ near the brane, which tends rapidly to zero, outside in the bulk. Note, that the nonzero value of the scalar field near the brane is stabilized by the interaction term $\lambda(\Phi \cdot \Phi)^{2}$.

Now will try to give a brief description of the new stable metric of the nonminimally coupled theory with negative $\xi$. In this case the new stable metric is expected to be of the form of Eq. (5) (or Eq. (10)). However, the behavior of the warp factor is not given by the equation $a(z)=e^{-k|z|}$ anymore. The exact form for $a(z)$ can be found only if we solve the system of the equations of motion for the lagrangian of the system (see Eqs. (6) and (2)) with two unknown functions $a(z)$ and $\Phi(z)$ and the appropriate boundary conditions. Note that as $\Phi(z) \rightarrow 0$ for $|z| \rightarrow+\infty$, the warp factor for $|z| \rightarrow+\infty$ must be of the form $a(z)=e^{-k|z|}$ or $a(z)=e^{k|z|}$, as these two functions satisfy the equations of motion if we set $\Phi(z)=0$. Otherwise in this paper we have not determined analytically or numerically the metric of the new stable solution, and we have left this problem for future investigation.

However, in order to estimate quantitatively the scalar field vacuum as a function of $\mathrm{z}$ we will assume that the RS-metric is fixed "by hand". We have solved numerically the equation of motion which corresponds to the Lagrangian of Eq. (6) for $\xi<0$, in the background of the RS-metric, and we have plotted our results in Fig. 1 for $z \geq 0$, as $\Phi(z)=\Phi(-z)$. We see that indeed the scalar field vacuum is nonzero on the brane and zero in the bulk, as expected from the behavior of Ricci scalar $\mathrm{R}$ versus $z$ (or $w$ ) (for details see the next section). In particular, we solved numerically the ordinary differential equation:

$$
-\Phi^{\prime \prime}(z)+4 k \Phi^{\prime}(z)+20 k^{2}|\xi| \Phi(z)+4 \lambda \Phi^{3}(z)=0
$$

for $z>0$, with the boundary conditions

$$
\partial_{z} \Phi(0)+8 k|\xi| \Phi(0)=0, \quad \Phi(+\infty)=0
$$

The first boundary condition comes from the $\delta(z)$ term in Riccy scalar R of Eq. (12) below. In addition, we emphasize that the potential term $\lambda(\Phi \cdot \Phi)^{2}$ is necessary in order to achieve a behavior like that of Fig. 1] 
In the framework of the above discussion we can argue in favor of a possible scenario for the gauge field localization on the RS2-brane world. The nonzero value ${ }^{3}$ of $\Phi_{3}$ field, for $z \approx 0$, implies a non-confinement phase on the brane (or the $\mathrm{SU}(2)$ symmetry is spontaneously broken to $\mathrm{U}(1)$ on the brane). On the other hand, the zero value of $\Phi_{3}$ outside the brane implies a confinement phase into the bulk, (or the $\mathrm{SU}(2)$ symmetry is restored in the bulk). In this way the gauge field of $\mathrm{U}(1)$ theory (photon) is localized on the brane, as for escaping in the bulk, it requires energy equal to $\Lambda_{\text {gap }}$, where $\Lambda_{\text {gap }}$ is the mass gap emerging from the nonperturbative confining dynamics of the $\mathrm{SU}(2)$ gauge field theory, in the bulk. Note, that in principle, the same mechanism can be used for a model with an arbitrary gauge group $G$ in the bulk which breaks to a subgroup $G^{\prime}$ on the brane. Similarly, the gauge fields related to $G^{\prime}$ are localized on the brane and are separated by a mass gap $\Lambda_{\text {gap }}$ from the bulk modes, which are massive because of confinement (also see Refs. [7, 14]).
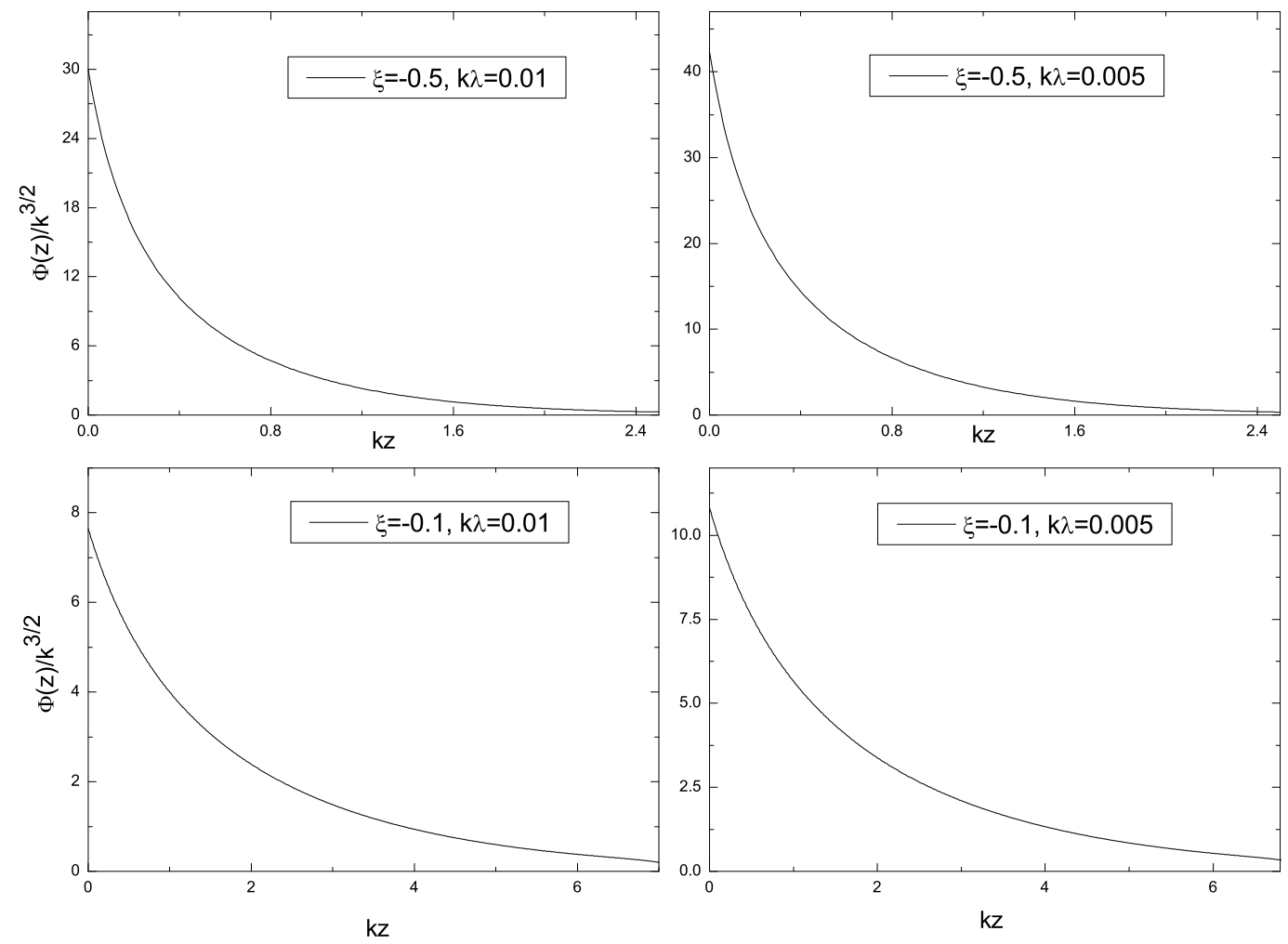

Figure 1: The scalar field vacuum $\Phi(z) / k^{3 / 2}$ versus $z(z>0$ as $\Phi(z)=\Phi(-z))$, for $k \lambda=$ $0.01,0.005$, and $\xi=-0.5,-0.1$, in the background of the RS-metric.

\footnotetext{
${ }^{3}$ We assume that the scalar field is directed toward the $\mathrm{c}=3$ direction in the isospin space.
} 


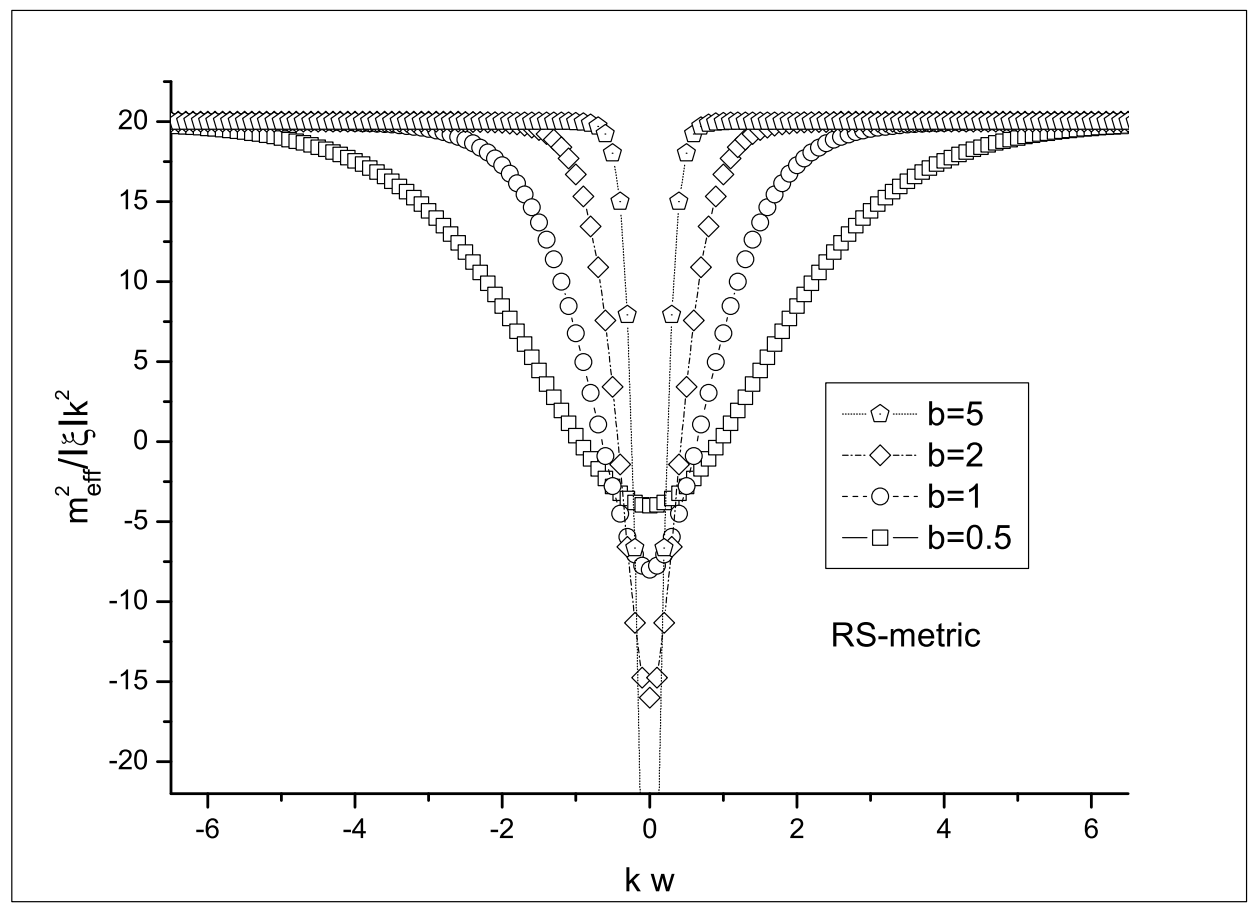

Figure 2: The effective mass $m_{\text {eff }}^{2}=-|\xi| R$ versus the extra dimension for the RSmetric of Eq. (8). In order to plot the delta function we used the representation $\delta(w)=\lim _{b \rightarrow+\infty} b \operatorname{sech}(b w)^{2}$.

\section{$3 \quad$ Ricci scalar as an effective $\mathrm{z}($ or $\mathrm{w})$-dependent mass term for the scalar field}

In this section we present an alternative way to establish qualitatively the picture of the nonvanishing $\Phi$-condensation near the brane, and the $\Phi=0$ in the bulk.

It is convenient to make the change of variable

$$
w=\operatorname{sgn}(z) \frac{\left(e^{k|z|}-1\right)}{k}
$$

Then the metric of Eq. (5) can be put into the manifestly conformal, to the five-dimensional Minkowski space, form

$$
d s^{2}=\alpha^{2}(w)\left(d x_{0}^{2}-d x_{1}^{2}-d x_{2}^{2}-d x_{3}^{2}-d w^{2}\right)
$$

where $\alpha(w)=1 /(k|w|+1)$

We can show that for the above metric of Eq. (10) the Ricci scalar is

$$
R(w)=-4\left(2 \frac{\alpha^{\prime \prime}(w)}{a^{3}(w)}+\frac{\left(\alpha^{\prime}(w)\right)^{2}}{\alpha^{4}(w)}\right)
$$


and for $\alpha(w)=1 /(k|w|+1)$ we obtain that

$$
R(w)=16 k \delta(w)-20 k^{2}
$$

Note the opposite signs of the delta function and the constant term in the above equation.
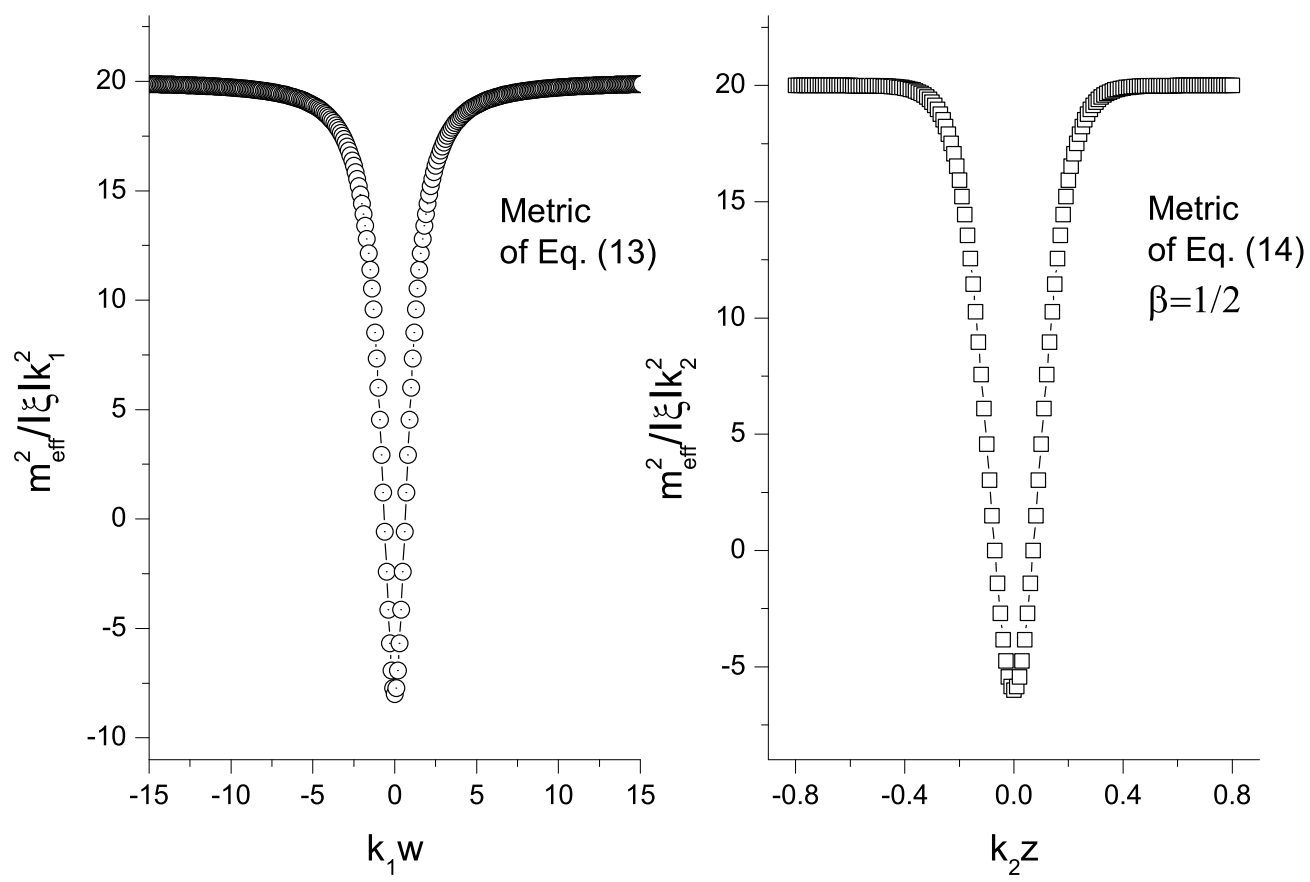

Figure 3: The effective mass $m_{\text {eff }}^{2}=-|\xi| R$ versus the extra dimension for the metrics of Eqs. (13) and (14).

From the lagrangian of Eq. (6) we see that the interaction term $-\xi R \Phi^{2}$, can be viewed as an effective w-dependent mass term, which corresponds to the scalar field $\Phi$ (for more details see Ref. [20] and references there in). We see that, for $\xi<0$, outside the brane the effective mass $m_{\text {eff }}^{2}(w)=-|\xi| R$ is positive (see Eq. (12)), and thus the $\mathrm{SU}(2)$ symmetry is not violated. On the other hand, for $w \approx 0$, the existence of the delta function renders the value of $m_{e f f}^{2}(w)$ negative on the brane (particularly $m_{e f f}^{2}(w)=-\infty$ on the brane), and thus the $\mathrm{SU}(2)$ symmetry is spontaneously broken.

As a figure with a delta function may cause same confusion, we will use the representation $\delta(w)=\lim _{b \rightarrow+\infty} b \operatorname{sech}(b w)^{2}$, in order to plot the delta function. In Fig. 2 we have plotted the effective mass $m_{e f f}^{2}(w) /|\xi| k^{2}$ versus $k w$, for several values of $b$. In this figure we see that $m_{\text {eff }}^{2}(w)$ is negative near the brane and positive in the bulk, as is expected.

It is worth to note that the mechanism of this paper is expected to work also for metrics other than that of Eq. (5). For this we have assumed the two metrics with smooth warp factors, of Eqs. (13) and (14). We see that for large values of the extra dimension both of them tend asymptotically to an $A d S_{5}$ spacetime. The metric of Eq. (13) has been found in 
Ref. 21] by M. Giovannini in the case of an additional Gauss-Bonnet term in the gravity action. The metric of Eq. (14) has been found in Ref. [5] by A. Kehagias and K. Tamvakis, in the case of a bounce type solution in the extra dimension (see their paper for a description of the parameters in Eq. (13)). Fig. 2 confirms that indeed the effective mass, for these two metrics, is negative on the brane and positive in the bulk, as is needed for the above presented mechanism to work.

$$
\begin{aligned}
& d s^{2}=\alpha^{2}(w)\left(d x_{0}^{2}-d x_{1}^{2}-d x_{2}^{2}-d x_{3}^{2}-d w^{2}\right), \quad \alpha(w)=\frac{1}{\sqrt{k_{1}^{2} w^{2}+1}} \\
& d s^{2}=a^{2}(z)\left(d x_{0}^{2}-d x_{1}^{2}-d x_{2}^{2}-d x_{3}^{2}\right)-d z^{2}, \quad a(z)=\frac{e^{-\beta \tanh ^{2}\left(k_{2} z\right)}}{\left(\cosh ^{2}\left(k_{2} z\right)\right)^{2 \beta}}
\end{aligned}
$$

\section{The tachyon mode for the scalar field}

In this section we study in detail the spectrum of the scalar field in the case of the additional interaction term $-\xi R \Phi^{2}$. We consider a small perturbation $\Phi_{3}$, directed toward the $c=3$

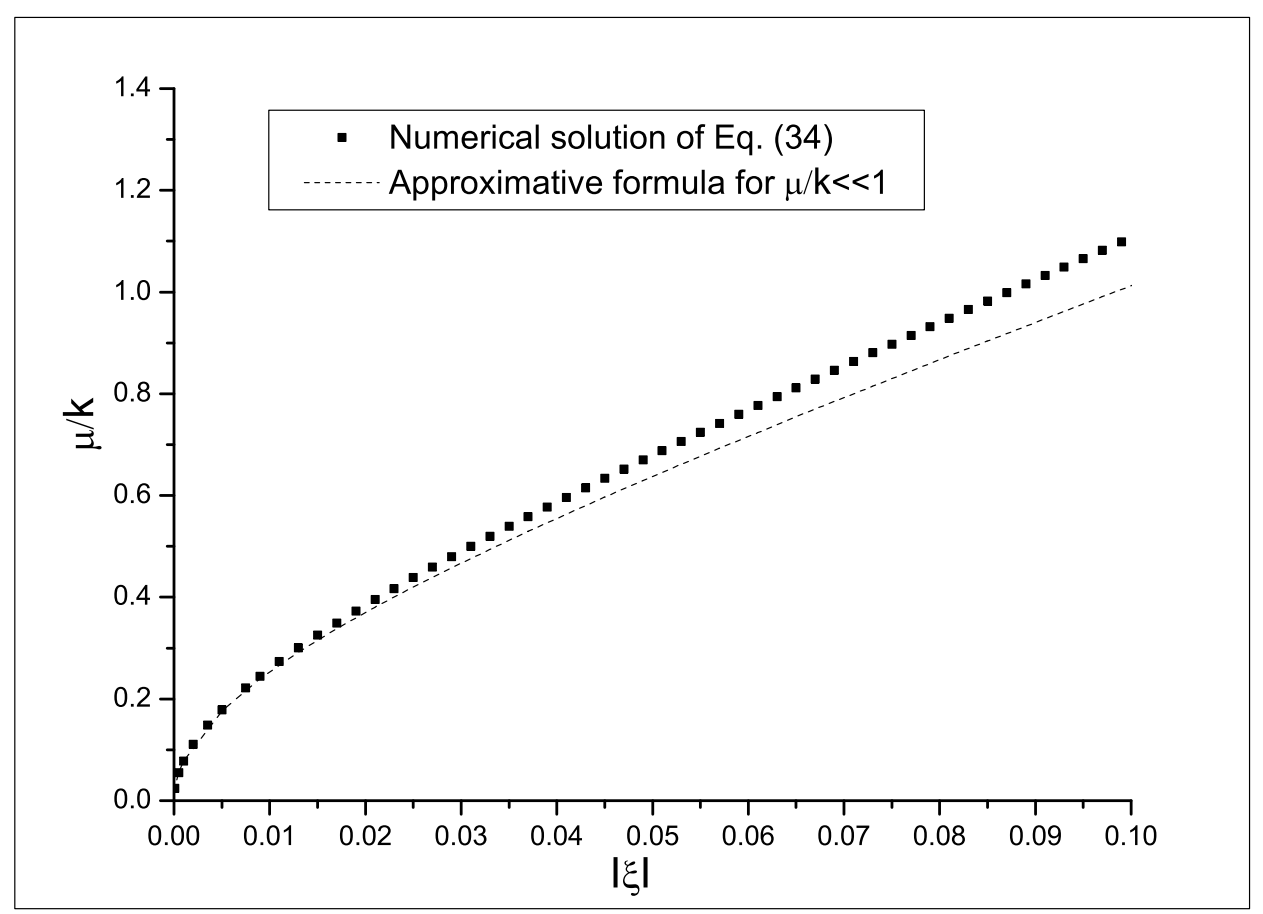

Figure 4: $\mu / k$ versus $\mathrm{k}$. The continuous line corresponds to the approximative formula of Eq. (35) for $\mu / k<<1$, and the discrete points to the exact solution of Eq. (34), as it is found numerically. We see that indeed the formula of Eq. (35) gives the correct result for $|\xi|<<1$.

direction in the isospin space, around the solution of Eq. (5). The corresponding linearized 
equation of motion is

$$
\frac{1}{\sqrt{|g|}} \partial_{M}\left[\sqrt{|g|} g^{M N} \partial_{N} \Phi(x, w)\right]+\xi R(w) \Phi(x, w)=0
$$

where, for the sake of simplicity, we have dropped the isospin index from the scalar field. In addition $g^{M N}=\left(1 / \alpha^{2}(w)\right)\left(\eta^{\mu \nu},-1\right)(\mu, \nu=0,1,2,3)$ where $\eta^{\mu \nu}=(1,-1,-1,-1)$, and $\sqrt{|g|}=\alpha^{5}(w)$. Eq. (15) can be written as

$$
\alpha^{3} \eta^{\mu \nu} \partial_{\mu} \partial_{\nu} \Phi-\partial_{w}\left(\alpha^{3} \partial_{w} \Phi\right)+\alpha^{5} \xi R \Phi=0
$$

We are looking for solutions of the form

$$
\Phi(x, w)=\phi(x) \frac{\psi(w)}{\alpha^{3 / 2}(w)}
$$

where

$$
\eta^{\mu \nu} \partial_{\mu} \partial_{\nu} \phi(x)=-m^{2} \phi(x)
$$

or $\phi(x) \sim e^{i p x}$, and $m^{2}=p_{\mu} p^{\mu}$ is the four dimensional mass.

The function $\psi(w)$ verifies the Schrondiger like equation

$$
-\psi^{\prime \prime}(w)+\left[V(w)-m^{2}\right] \psi(w)=0
$$

where the potential $V(w)$ is defined as

$$
V(w)=\frac{\left(\alpha^{3 / 2}(w)\right)^{\prime \prime}}{\alpha^{3 / 2}(w)}+\xi \alpha^{2}(w) R(w)
$$

Note that Eq. (19) determines the four dimensional particle spectrum of the theory.

For the metric of Eq. (10) we obtain

$$
\frac{\left(\alpha^{3 / 2}(w)\right)^{\prime \prime}}{\alpha^{3 / 2}(w)}=-3 k \delta(w)+\frac{15}{4} k^{2} \frac{1}{(k|w|+1)^{2}}
$$

Thus from Eqs. (12), (20) and (21) we obtain

$$
V(w)=-k(3-16 \xi) \delta(w)+k^{2}\left(\frac{15}{4}-20 \xi\right) \frac{1}{(k|w|+1)^{2}}
$$

If we set $y=k w$ we obtain

$$
-\psi^{\prime \prime}(y)+\left[\bar{V}(y)-\frac{m^{2}}{k^{2}}\right] \psi(y)=0
$$

The boundary conditions on the brane are

$$
\begin{aligned}
\psi(+0)-\psi(-0) & =0 \\
\partial_{y} \psi(+0)-\partial_{y} \psi(-0) & =-b \psi(0)
\end{aligned}
$$


where the second condition arises due to the existence of the delta function in Eq. (22) (or Eq. (29)). For the definition of b see Eq. (27) below. The potential $\bar{V}(y)$ is given by Eq. (29) below.

As we have emphasized in previous sections we expect a tachyon mode for $\xi<0$ (or $|\xi|=-\xi)$. If we set $m^{2}=-\mu^{2}<0$ we obtain.

$$
-\psi^{\prime \prime}(y)+\left[\bar{V}(y)+\frac{\mu^{2}}{k^{2}}\right] \psi(y)=0
$$

In addition, if we set

$$
\begin{aligned}
& b=(3+16|\xi|) \\
& c=\left(\frac{15}{4}+20|\xi|\right)
\end{aligned}
$$

the potential $V(y)$ can be written in the form

$$
\bar{V}(y)=-b \delta(y)+\frac{c}{(|y|+1)^{2}}
$$

If we search for a normalizable solution of Eq. (26) we obtain that

$$
\begin{aligned}
& \psi(y)=N \sqrt{y+1} K_{\nu}\left[\frac{\mu}{k}(y+1)\right] \quad \text { if } \quad y \geq 0 \\
& \psi(y)=N \sqrt{-y+1} K_{\nu}\left[\frac{\mu}{k}(-y+1)\right] \quad \text { if } \quad y \leq 0
\end{aligned}
$$

where $K_{\nu}(x)$ are the modified Hankel functions, and $\nu=\sqrt{c+\frac{1}{4}}=\sqrt{4+20|\xi|}$.

Note that if take into account Eq. (9), then

$$
\widetilde{\psi}(z)=N e^{\frac{k|z|}{2}} K_{\nu}\left[\frac{\mu}{k} e^{k|z|}\right]
$$

From the boundary condition (25), if we take into account Eqs. (30) and (31) we obtain

$$
(b+1) K_{\nu}\left(\frac{\mu}{k}\right)+2 \frac{\mu}{k} K_{\nu}^{\prime}\left(\frac{\mu}{k}\right)=0
$$

If we use the identity $K_{\nu}^{\prime}(x)=-K_{\nu-1}(x)-\frac{\nu}{x} K_{\nu}(x)$ we obtain

$$
(b+1-2 \nu) \frac{K_{\nu}\left(\frac{\mu}{k}\right)}{\left(\frac{\mu}{k}\right) K_{\nu-1}\left(\frac{\mu}{k}\right)}=2
$$

For $\mu<<k$, if we take into account the asymptotic formula $K_{\nu}(x) \approx \frac{1}{2} \Gamma(\nu)\left(\frac{2}{x}\right)^{\nu}$ as $x \rightarrow 0$, we find the solution

$$
\mu^{2}=4 k^{2}(1+4|\xi|-\sqrt{1+5|\xi|})(2 \sqrt{1+5|\xi|}-1)
$$

and for $|\xi|<<1$ we find the simple expression

$$
\mu^{2} \approx k^{2}\left(6|\xi|+\frac{85}{2} \xi^{2}\right)
$$


Note that for $\xi=0$ we obtain $\mu=0$, as is expected.

In Fig. 4 we compare the approximative formula of Eq. (35) with the exact solution of Eq. (34), as it is found by a numerical method. We see that indeed Eq. (35) gives the correct result for $|\xi|<<1$ (or for $\mu / k<<1$ ). In addition, we have checked numerically that Eq. (34) has a unique solution for all the range of values of $\xi<0$, which is a monotonic increasing function of $|\xi|$. However, if $|\xi|$ is not small $(|\xi|>>0.04$ and $\mu / k>>0.6)$ as we see in Fig. 3) Eq. (35) fails to give the correct value for $\mu / k$. For $\xi>0$ there is no tachyon mode of the form of Eq. (32). Finally, we note that the case of $\xi>0$ may have same interest, however it is not investigated in this paper.

\section{Conclusions}

We investigated the spectrum of a scalar field, with a possible interaction term of the form $-\xi R \Phi^{2}$, in the background of the RS-metric. We show that the zero mode for $\xi=0$ turns into a tachyon mode, in the case of a nonzero negative value of $\xi(\xi<0)$. As we argue in sections 2,3 and 4, we expect that the tachyon mode renders the classical solution of Eq. (5) (RS-metric plus $\Phi=0$ ) unstable, against a new stable solution with nonzero $\Phi$-field condensation near the brane, and a new metric of the form of Eq. (5) with a different warp factor (for details see section 2).

In the framework of the above discussion we can construct a simple model where the Dvali-Shifman mechanism is triggered by the geometry of the multidimensional space time. The advantage of this model is that the neutral scalar field in Ref. 2], which forms a kink topological defect towards the extra dimension, is not necessary, as it has been replaced by the RS-metric.

\section{Acknowledgements}

We are grateful to Professors A. Kehagias, G. Koutsoumbas and N. Tetradis for reading and commenting on the manuscript. K.F thanks Professor M. Giovannini for useful and elaborating discussions during his visit at CERN. P.P. thanks Dr. P. Manouselis for impor-

tant discussions. This work was partially supported by "Thales" project of NTUA and the "Pythagoras" project of the Greek Ministry of Education-European community (EPEAEKEKT, 25/75).

\section{References}

[1] L. Randall and R. Sundrum, Phys. Rev. Lett. 83 (1999) 3370; L. Randall and R. Sundrum, Phys. Rev. Lett. 83 (1999) 4690;

[2] G. Dvali and M. Shifman, Phys. Lett. B 396 (1997) 64.

[3] B. Bajc and G. Gabadadze, Phys. Lett. B 474 (2000) 282; I. Oda, Phys. Lett. B 496 (2000) 113. 
[4] V. A. Rubakov, Phys. Usp. 44 (2001) 871; S. L. Dubovsky and V. A. Rubakov, Int. J. Mod. Phys. A 16 (2001) 4331.

[5] A. Kehagias and K. Tamvakis, Phys. Lett. B 504 (2001) 38

[6] A. Neronov, Phys. Rev. D 64 (2001) 044018; M. Giovannini, Phys. Rev. D 66 (2002) 044018; A. Pomarol Phys. Lett. B 486 (2000) 153; H. Davoulias, J.L. Hewett and T.G. Rizzo, Phys. Lett. B 473 (2000) 43.

[7] M. Laine, H.B. Meyer, K. Rummukainen, M. Shaposnikov, JHEP 0404 (2004) 027.

[8] N. Arkani-Hamed and M. Schmaltz, Phys. Lett. B 450 (1999), 92.

[9] N. Tetradis, Phys. Lett. B 479 (2000) 265.

[10] D. A. Demir and M. Shifman, Phys. Rev. D 65 (2002) 104002.

[11] Y.K. Fu and H.B. Nielsen, Nucl. Phys. B 236 (1984), 167.

[12] C.P. Korthals-Altes, S. Nicolis and J. Prades, Phys. Lett. B316 (1993), 339; A. Hulsebos, C.P. Korthals-Altes and S. Nicolis, Nucl. Phys. B450 (1995) 437.

[13] P. Dimopoulos, K. Farakos, A. Kehagias and G. Koutsoumbas, Nucl. Phys. B 617 (2001) 237-252.

[14] P. Dimopoulos, K. Farakos, G. Koutsoumbas, Phys. Rev. D65: (2002), 074505.

[15] P. Dimopoulos, K. Farakos, G. Koutsoumbas, C.P. Korthals-Altes, S. Nicolis, JHEP02(005) 2001; K. Farakos, G. Koutsoumbas, S. Nicolis, Eur. Phys. J C24 (2002), 287-296; P. Dimopoulos, K. Farakos, Phys. Rev. D70 (2004) 045005.

[16] N. D. Birrell, P. C. W. Davies, Quantum fields in curved space, Camprige University Press 1982.

[17] S. M. Carroll, Spacetime and geometry, Addison Wesley 2004.

[18] A. D. Dolgov, The very early universe, edited by G. W. Gibbons, S. W. Hawking and S. T. C. Siklos, (Camprige University Press, Camprige 1973). A. Dolgov and D. N. Pelliccia, Scalar field instabilitty in de Sitter space -time, hep-th/0502179.

[19] L. H. Ford, Phys. Rev. D 35 (1987) 2339;

[20] J. Gu and W. P. Hwang, Mod.Phys.Lett. A17 (2002) 1979-1990.

[21] M. Giovannini, Phys. Rev. D 64 (2001) 124004. 